\title{
Effects of wind turbine wake on atmospheric sound propagation
}

\author{
Barlas, Emre; Zhu, Wei Jun; Shen, Wen Zhong; Kelly, Mark C.; Andersen, Søren Juhl
}

Published in:

Applied Acoustics

Link to article, DOI:

10.1016/j.apacoust.2017.02.010

Publication date:

2017

Document Version

Peer reviewed version

Link back to DTU Orbit

Citation (APA):

Barlas, E., Zhu, W. J., Shen, W. Z., Kelly, M. C., \& Andersen, S. J. (2017). Effects of wind turbine wake on atmospheric sound propagation. Applied Acoustics, 122, 51-61. https://doi.org/10.1016/j.apacoust.2017.02.010

\section{General rights}

Copyright and moral rights for the publications made accessible in the public portal are retained by the authors and/or other copyright owners and it is a condition of accessing publications that users recognise and abide by the legal requirements associated with these rights.

- Users may download and print one copy of any publication from the public portal for the purpose of private study or research.

- You may not further distribute the material or use it for any profit-making activity or commercial gain

- You may freely distribute the URL identifying the publication in the public portal 


\title{
Effects of Wind Turbine Wake on Atmospheric Sound Propagation
}

\author{
Emre Barlas, Wei Jun Zhu, Wen Zhong Shen, Mark Kelly, Søren Juhl Andersen \\ Department of Wind Energy, Technical University of Denmark, 2800 Kongens Lyngby, Denmark
}

\begin{abstract}
In this paper, we investigate the sound propagation from a wind turbine considering the effects of wake-induced velocity deficit and turbulence. In order to address this issue, an advanced approach was developed in which both scalar and vector parabolic equations in two dimensions are solved. Flow field input was obtained using the actuator line (AL) technique with Large Eddy Simulation (LES) to model the wind turbine and its wake and from an analytical wake model. The effect of incoming wind speed and atmospheric stability was investigated with the analytical wake input using a single point source. Unsteady acoustic simulations were carried out with the AL/LES input for three cases with different incoming turbulence intensity, and a moving source approach to mimic the rotating turbine blades. The results show a non-negligible effect of the wake on far-field noise prediction. Particularly under stable atmospheric conditions, SPL amplification reaches up to $7.5 \mathrm{~dB}$ at the wake centre. Furthermore, it was observed that when the turbulence intensity level of the incoming flow is higher, the SPL difference between the moving and the steady source is lower.
\end{abstract}

Keywords: Atmospheric acoustics, Vector parabolic equation, Wind turbine sound, Wind turbine wake

\section{Introduction}

Noise is one of the main obstacles achieving a broader public acceptance of wind energy. In order to reduce the transmission cost, wind farms are often placed close to populated areas. This demands accurate noise prediction tools and noise reduction techniques which are difficult tasks due to the complexity of wind turbine noise generation and propagation mechanisms. Turbines emit time dependent sound that varies with the incoming flow (i.e. wind speed and turbulence levels), and the turbine design and operation conditions (i.e. rotational speed and rotor design). Moreover, downwind propagation of the emitted sound is influenced by the turbine induced velocity deficit and turbulence. This issue is addressed by Heimann et al. (2011) with a ray-based sound particle method where the flow field input is obtained either from full scale experiments or meteorological micro scale models. The study considers a single night case with a temperature inversion and a low-level jet whose wind speed maximum is below the rotor plane crest. Depending on the meteorological model used for the flow field input method the results show variations (overall sound pressure levels are either higher or lower than the case without the wake deficit) in the near field (up to $700 \mathrm{~m}$ ). The far field noise $(700 \mathrm{~m}-1000$ $\mathrm{m}$ ) is found to be consistently higher for all simulations when the wake is taken into account. Lee et al. (2015) uses parabolic equation method for the acoustic simulations and a Reynolds Averaged Navier Stokes based solver for the flow field input. Different to Heimann et al. (2011) the simulations were carried out for four incoming flow wind shear values and the computational domain extends up to $3000 \mathrm{~m}$ in the streamwise direction. The results show considerable variations depending on the incoming wind profile. They found that for a small wind shear case, the wake flow tends to increase the far-field noise levels while the opposite is observed for much higher wind shear values.

The present work employs an approach by coupling the high resolution Actuator Line (AL)/Large Eddy Simulation (LES) solutions with the Parabolic Equation (PE) method in order to investigate the unsteady downwind sound propagation in the wake of a wind turbine. Using the time dependent flow input, frequency dependent acoustic simulations are carried out successively. Three different incoming turbulence intensity cases are simulated with AL/LES, each yielding a different velocity distribution thus changing the refraction patterns of sound waves. Additionally the superposition of various incoming wind profiles with analytical wake models is also used for flow field input. Thereby, comparative studies are carried out to assess the need for unsteady turbulent flow input. Another comparison is carried out with two types of source modelling. The conventional approach is to represent the wind turbine with a single point source located at hub height. In this study an unsteady approach was used to mimic the rotation of the wind turbine blades. Thereby, the amplitude modulation due to blade passage is captured. The main objectives of the study are grasping a better understanding of the relationship between wake dynamics and far field noise levels as well as assessing the influence of turbulent flow input and source modelling for wind turbine sound propagation.

The structure of the paper is as following; first the computational models used in this study are described for both acoustic and flow simulations, respectively. Then the results are discussed in four different subsections. First part is the validation and grid independence study for the PE method. In the second part single PE simulations are carried out with the flow input 
obtained from the analytical wake model superposed with various wind profiles. This part is called the steady case. The third part uses the flow input obtained from LES and referred to as the unsteady case. The fourth part deals with comparison of the aforementioned two methods and finally a discussion and a conclusion sections are included.

\section{Computational Models}

\subsection{Models for Sound Propagation}

Wind turbines significantly modify the flow behind them, hence a sound propagation method that can handle the range dependent flow field (varying along the propagation path) is required. On the other hand, distances on the order of 2-3 $\mathrm{km}$ restricts the usage of computationally demanding time domain methods. Therefore, a two-dimensional Parabolic Equation (PE) method was chosen as a compromise between accuracy and computational resources.

Following the notation in Blanc-Benon et al. (2001) the PE method can be divided into two groups, namely scalar and vector. The former is the conventional approach where the moving atmosphere is replaced by a hypothetical motionless medium with the effective sound speed $c_{e f f}=c+v_{x}$, where $v_{x}$ is the wind velocity component along the direction of propagation between source and receiver. In the latter approach, the vector properties of the velocity field are maintained. Thus the equation contains new terms. The scalar PE method is expected to perform well when the source and receiver are close to the ground, as the sound propagation direction is nearly horizontal and the scattering angle (angle between the sound wave and the wave scattered by turbulence) do not exceed a certain limit. For both cases the derivation can be carried out starting from the equation for the sound field $P^{\prime}$ in inhomogeneous moving medium Ostashev et al. (1997) (here given with uniform density assumption);

$$
\left[\nabla^{2}+k^{2}(1+\epsilon)-\frac{2 i}{\omega} \frac{\partial v_{i}}{\partial x_{j}} \frac{\partial^{2}}{\partial x_{i} \partial x_{j}}+\frac{2 i k}{c_{0}} \mathbf{v} \cdot \nabla\right] P^{\prime}(r)=0
$$

where $k=\omega / c_{0}$ ( $\omega$ is the radian frequency of the sound, $c_{0}$ is the reference speed of sound), $P^{\prime}(r)$ is the monochromatic sound field, $\epsilon=\left(c_{0} / c\right)^{2}-1$. Note that, Eq. 1 reduces to a classical Helmholtz equation if $\mathbf{v}=0 ;\left[\nabla^{2}+k^{2}(1+\epsilon)\right] P^{\prime}(r)=0$. Further mathematical manipulation in order to reduce these equations to a one way parabolic equation is carried out in Ostashev et al. (1997). If the derivation starts from Eq. 1 and maintains the terms with wind velocity then it reduces to Turbulent-Wind Wide Angle Parabolic Equation (TW-WAPE) and it is also referred to as the vector PE. If further manipulation is carried out by setting all velocities to zero, then it reduces to Wide Angle Parabolic Equation (WAPE) and it is referred to as the scalar PE. In this study both scalar and vector PE methods are used. The aim is to test and present the limitations of WAPE in comparison to TW-WAPE for an elevated source such as wind turbine, with range dependent velocity profiles. For both methods a semi-implicit marching scheme is used, with central differences in the vertical direction $(z)$ and the Crank-Nicolson method in the horizontal direction $(x)$. These two PE methods yield either tri-diagonal (WAPE) or penta-diagonal matrices (TW-WAPE) to be solved at each space step. The spatial resolution for both directions is set to one-eighth of the wavelength $(\Delta x=\Delta z=\lambda / 8)$; where $\lambda$ is the wavelength of the solving frequency. Only flat terrain is considered and the ground impedance was characterized using the Delany-Bazley model Delany and Bazley (1970). An effective flow resistivity value of $200 \mathrm{kPa} \mathrm{s} / \mathrm{m}^{2}$ was chosen, as this is a typical value for grassland where onshore turbines are commonly located.

The PE methods require a starter function which essentially represents the sound source. In this study the starter function derived in West et al. (1992) is used. One approach to mimic the dynamics of the wind turbine sound is treating the wind turbine to be composed of lumped sources located near the tips, i.e., at $85 \%$ of the blade span. This approach is a result of the source location studies carried out by Oerlemans et al. (2007) which states that the sound is produced in the outer part of the blades. Considering a wind turbine rotating in and out of 2D PE plane, three separate simulations are carried out for each atmospheric condition. For each simulation the single point source is located either at top tip height $(114 \mathrm{~m})$ or hub height $(80 \mathrm{~m})$ or bottom tip height $(46 \mathrm{~m})$. This set of simulations is referred to as the steady case. The unsteady case employs a moving source approach. The main difference between steady and unsteady cases is that the flow field input for each PE simulation is updated every $0.11 \mathrm{~s}$ for the unsteady case and at each time step a point source is translated either up or down taking the turbine rotational speed, the diameter and the hub height into account (see Sec. 2.2.2). Since the focus of this study is the flow field effects on propagation, the source power levels are kept constant for all simulations.

\subsection{Models for the Flow Field Input}

The PE methods described in the preceding section need flow field input, either as scalar or vector. These profiles were obtained with two different methods that are elaborated in the following subsections.

\subsubsection{Case with Analytical Wake Method}

In the steady case the streamwise velocity profile is obtained using the analytical wake model proposed by Bastankhah and Porte-Agel (2014). The model uses the mass and momentum conservation by neglecting the viscous and pressure terms and then applies it on the control volume around the turbine. Subsequently, a Gaussian profile in vertical is proposed for the velocity deficit in the wake and an expression for the normalized wind speed deficit is given. The required inputs to the model are hub height $\left(z_{h}\right)$, turbine diameter $(D)$ and thrust coefficient $\left(C_{t}\right)$ which are selected to be $z_{h}=80 \mathrm{~m}, D=80 \mathrm{~m}$ and $C_{t}=0.8$. Additionally, a tunable parameter $\left(\mathrm{k}^{*}\right)$ for the wake growth rate is necessary. This parameter is adjusted with respect to atmospheric stability via an LES study by Abkar and Porte-Agel (2014) as reference.

The ambient mean flow and temperature profiles are obtained using the Monin-Obukhov similarity theory (MOST) for three 
different stability conditions. The expressions used for the profiles in this study can be found in Salomons (2001). The relevant parameters are given in Table 1, with the inverse Obukhov length $\left(L_{M O}^{-1}\right)^{1}$ and all simulations are carried out with a aerodynamic roughness length $z_{0}=0.1 \mathrm{~m}$.

\begin{tabular}{lccc}
\hline ABL Classes & $L_{M O}^{-1}[\mathrm{~m}]$ & $u_{*}[\mathrm{~m} / \mathrm{s}]$ & $\theta *[\mathrm{~K}]$ \\
\hline Stable (SBL) & 0.067 & 0.31 & 0.016 \\
Neutral (NBL) & 0 & 0.4 & 0 \\
Convective (CBL) & -0.067 & 0.46 & -0.044
\end{tabular}

Table 1: Surface-layer parameters used in the wind profile, for atmospheric stability classes considered here.

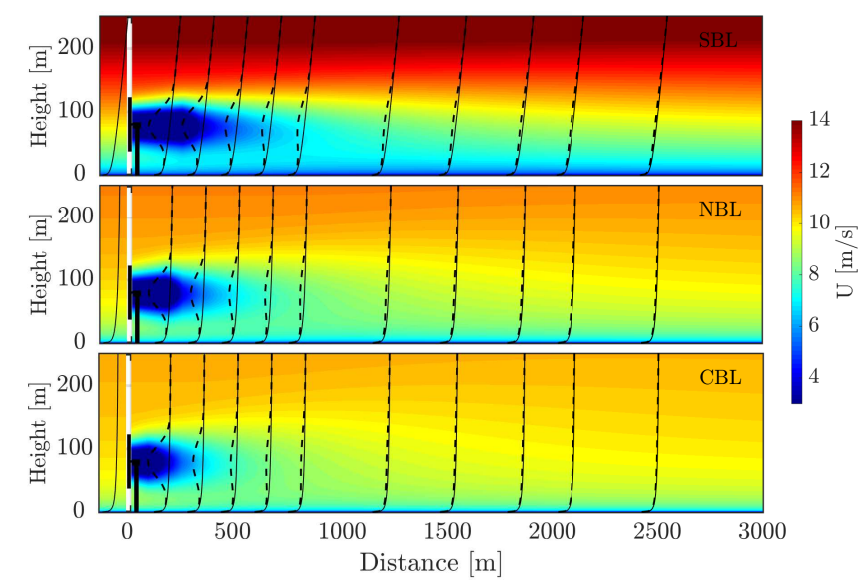

Figure 1: Contours of streamwise velocity in the middle vertical plane for different stability conditions. The flow fields are obtained from the analytical model in Bastankhah and Porte-Agel (2014). Top: Stable conditions $\mathrm{k}^{*}=0.02$ Middle: Neutral conditions $\mathrm{k}^{*}=0.03$ Bottom: Unstable conditions $\mathrm{k}^{*}=0.04$. Other relevant parameters are given in the text. Solid Line: Incoming wind profile. Dashed Line: Wake deficit wind profile

The flow fields obtained via superposing the wake deficits and the upstream wind profiles under three different atmospheric stability conditions are depicted in Fig. 1. Note that, for the case with the analytical wake input only streamwise velocity is used while the vertical velocity is set to zero for the vector PE method. Two things are worthwhile to point out regarding these flow fields: 1) increased stability yields a longer wake; 2) incoming wind profiles are different. In Sec. 3.2.2 these effects on refraction will be discussed in detail.

\subsubsection{Case with Large Eddy Simulation}

Flow fields are obtained from high resolution LES data for the unsteady case. The simulations are performed using the 3D flow solver EllipSys3D, which was developed as a collaboration between Technical University of Denmark (Michelsen (1992)) and Risø National Laboratory (Sørensen (1995)). EllipSys3D solves the discretised incompressible Navier-Stokes equations

\footnotetext{
${ }^{1}$ In contrast to $L_{M O}$, its inverse $L_{M O}^{-1}$ is amenable to statistical characterization Kelly and Gryning (2010).
}

in general curvilinear coordinates using a block structured finite volume approach in primitive variables. The influence of the wind turbine is simulated using the Actuator Line technique (for further details see Sørensen and Shen (2002)), which imposes body forces along the rotating lines. The body forces are calculated through a full aero-elastic coupling with Flex5, which computes the aerodynamic loads, see Øye (1996) for details on Flex5 and Sørensen et al. (2015) for details on the coupling. The fully coupled simulations also includes a controller, which means the turbine behaves as a real turbine and adjusts according to the incoming turbulent flow field. The modelled turbine is an upscaled version of the NM80 turbine based on the original NM80 (see Aagaard Madsen et al. (2010)) and the turbine is proprietary to Vestas Wind Systems A/S. The blade radius is $R=40 \mathrm{~m}$ and rated power is $2.75 \mathrm{MW}$ at rated wind speed of $14 \mathrm{~m} / \mathrm{s}$. The computational domain used for this study is [40 D X $10 \mathrm{D}$ X $10 \mathrm{D}]$ in the streamwise, vertical and lateral directions, respectively (D represents the turbine diameter, 80 $\mathrm{m}$ ) and the turbine is located $400 \mathrm{~m}$ from the inlet. The spatial resolution is $2.5 \mathrm{~m}$ in all directions and the computations were carried out for approximately 90 minutes of real time. The velocity perturbations upstream of the turbine are obtained from a pre-generated turbulent wind field (see Mann (1998)). The magnitude of the fluctuations were scaled in order to mimic three different incoming turbulence intensity $(0 \%, 3 \%, 10 \%)$. Out of 90 minutes long flow simulations, two independent 10 minutes long data are used for each turbulence intensity case to feed into the acoustic simulations. Both vertical and streamwise velocity components are extracted at $10 \mathrm{~Hz}$ and subsequently fed into the acoustic simulations. Instantaneous snapshots of the flow fields are depicted in Fig. 2. It can be seen that the turbine wake becomes less stable and persistent when incoming turbulence intensity increases as the inflow turbulence assists in breaking down the wake (Sørensen et al. (2015)). This will influence the sound propagation as will be shown later.

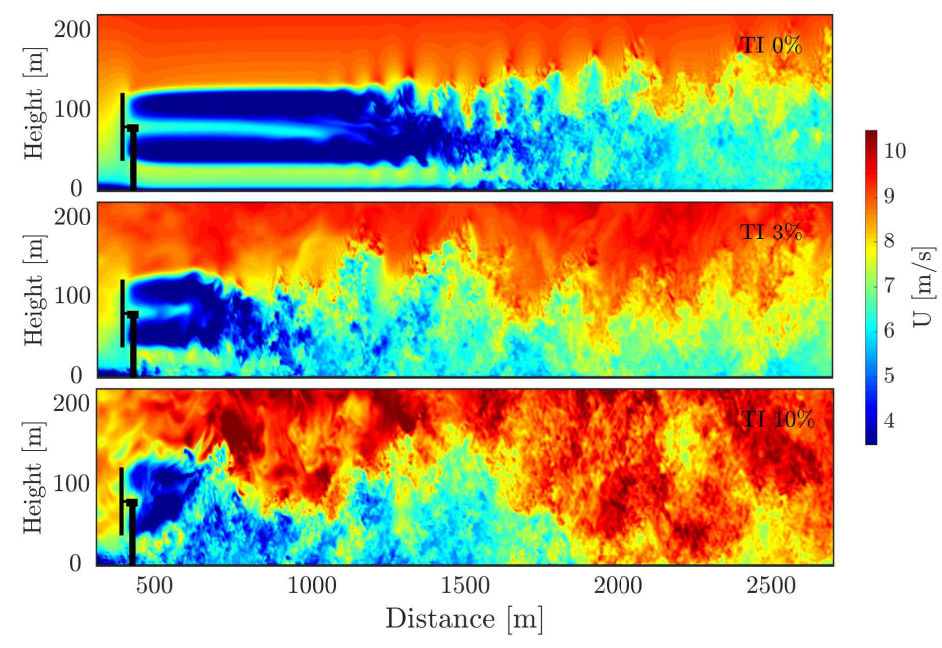

Figure 2: Contours of instantaneous streamwise velocity $(u)$ in the middle vertical plane for different incoming turbulence intensity $\left(\frac{u^{\prime}}{\bar{U}}\right)$. The flow fields are obtained from LES-AL technique. From top to bottom: $\mathrm{TI}=0 \%, \mathrm{TI}=3 \%$, $\mathrm{TI}=10 \%$ 


\section{Results}

All simulations are carried out for $1 / 3$-octave band with lower, centre and upper limit frequencies up to $630 \mathrm{~Hz}$ and summed logarithmically;

$$
L_{p(\text { sum })}=10 \log _{10}\left(\sum_{i=1}^{N} 10^{L p\left(f_{i}\right) / 10}\right)
$$

where $N$ is the number of frequencies used and $L_{p}\left(f_{i}\right)$ is the sound pressure level defined as;

$$
L_{p}\left(f_{i}\right)=L_{W-A}\left(f_{i}\right)-10 \log _{10} 4 \pi R^{2}-\alpha R+\Delta L
$$

where the A-weighted source power level for a wind turbine $\left(L_{W-A}\right)$ is obtained from the semi empirical source model Zhu et al. (2005), the second term on the right hand side stands for geometrical spreading, the third term represents the atmospheric absorption where the absorption coefficient is calculated according to ISO $9613-1$ for air at $20^{\circ} \mathrm{C}$ with $80 \%$ relative humidity. The last term is the relative sound pressure level $\Delta L=20 \log _{10}\left(\left|P^{\prime}\right| /\left|P_{f}\right|\right)$ that represents the deviation from the free field of a source due to ground effect, atmospheric refraction, turbulence etc.

\subsection{Validation and Grid Independence Study}

The output from WAPE and TW-WAPE are compared to the work of Dallois et al. (2001). Fig. 3 shows the result for 680 $\mathrm{Hz}$, where the source is located at $2 \mathrm{~m}$ height and a receiver at $10 \mathrm{~m}$ height. It is seen that with the implementation used in this paper the phase shifts as well as the dips and peaks of SPL are captured. This validates the implementation of these two methods.
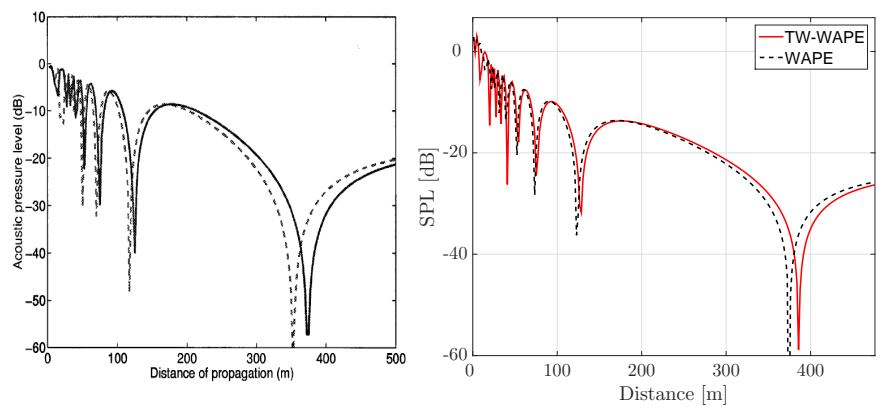

Figure 3: Validation case. Sound pressure levels for a receiver at $10 \mathrm{~m}$ height Left: Taken from Dallois et al. (2001) Right: Current work.

Furthermore, an error analysis is carried out in order to test how often the solution matrices should be updated in the space marching scheme for a range dependent flow field. This is done by running one benchmark case with both methods where the update is at each space step, $\Delta x$ (where $\Delta x=\lambda / 8$ ). Afterwards the error is quantified for cases with space steps of $5,10,50$ and $100 \Delta x$ by subtracting the relative sound pressure levels from the case with $\Delta x$. The highest error is detected under stable atmospheric conditions and Fig. 4 shows the evolution with distance for a receiver at $2 \mathrm{~m}$. It is clear that if the step is chosen much larger than the wavelength, i.e. 50 or $100 \Delta x$, the committed error is considerably high for TW-WAPE. A similar trend is observed for WAPE, however the error margin is much smaller. For both methods the error is less than $0.5 \mathrm{~dB}$ if the simulations with $5 \Delta x$ is used. Thus from this point on the update step is set to $5 \Delta x$.

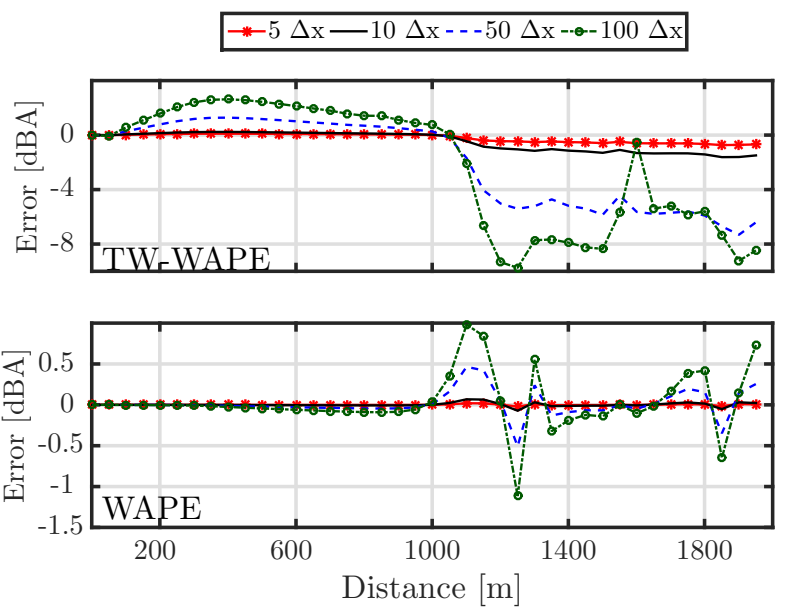

Figure 4: Sound pressure levels for a receiver at $2 \mathrm{~m}$ height under stable atmospheric conditions. The error is quantified by subtracting results from the benchmark case which is the case where the flow field update is at each space step Top: TW-WAPE Bottom: WAPE

\subsection{Steady Case with Analytical Wake Model}

\subsubsection{Effect of Wake under Neutral Stability Conditions}

The next step is to assess the difference between range dependent and independent profiles on sound propagation (also denoted with and without wake, respectively).

First, the case with source height at $80 \mathrm{~m}$ (hub height) is studied and an interesting refraction phenomenon is captured. Fig. 5 helps to give a visual understanding of this phenomenon. Considerably higher sound pressure levels are observed after certain distance from the source. This was also reported in Heimann et al. (2011) and Lee et al. (2015). The wake deficit and its downstream evolution are the key parameters which cause different refraction patterns of sound waves. Subsequently downwards bursting zones are generated (similar to ducting of sound waves near the ground observed in e.g. Wilson et al. (2003) ). These are the regions with high sound pressure level due to turbine induced velocity gradients. Note that in the wake, the mean wind speed vertical gradient $(\partial U / \partial z)$ at the top tip height is significantly greater than at the bottom tip, thus creating a more severe downward refraction than the upward refraction. Moreover, increased incoming hub height wind speed $\left(U_{h}\right)$ also yields stronger gradients. Thus the regions with higher sound pressure levels are widened. Two distinctly separated peak regions are detected for the case $U_{h}=14$ $\mathrm{m} / \mathrm{s}$. The first peak is caused by initial downward refraction. The second peak is due to firstly upward and afterwards downward refracted sound waves. 
Overall, the SPL differences due to the wake can reach up to $12 \mathrm{~dB}$ at certain ranges for a receiver at $2 \mathrm{~m}$ height as shown in Fig. 6. Note that the simulations with range independent profiles (without wake) yield higher sound pressure levels for the ranges before the bursting zones, namely before $1 \mathrm{~km}$. Even though, only one case without wake is shown $\left(U_{h}=6 \mathrm{~m} / \mathrm{s}\right)$ in this figure, this observation is valid for higher incoming wind speeds. The reason for this is that before the wake breakdown the acoustic waves are retained within the wake deficit region thus they do not reach to the ground. This results in attenuation of SPL in the near field (less than $1000 \mathrm{~m}$ ). The refraction patterns vary depending on the source height. This is studied in Sec. 3.2 .3

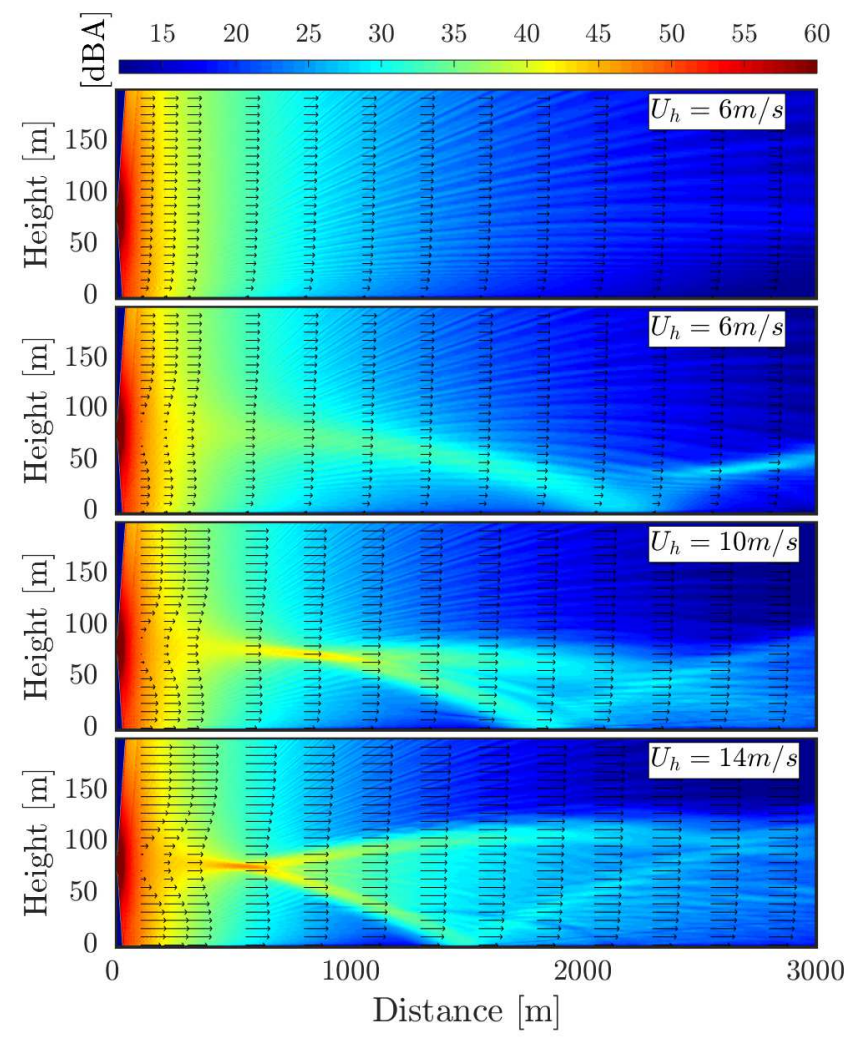

Figure 5: Contours of sound pressure levels calculated with TW-WAPE under neutral atmospheric stability conditions for various incoming hub height wind speeds. The velocity vectors are embedded in the figure. From top to bottom: Case without wake $U_{h}=6 \mathrm{~m} / \mathrm{s}$, case with wake $U_{h}=6 \mathrm{~m} / \mathrm{s}$, case with wake $U_{h}=10 \mathrm{~m} / \mathrm{s}$ and case with wake $U_{h}=14 \mathrm{~m} / \mathrm{s}$.

\subsubsection{Effect of Atmospheric Stability Conditions}

The incoming flow as well as the wake evolution is different for various atmospheric stability conditions. Wake recovery takes places differently due to varying mixing characteristics. For sound propagation this means that the refraction regions will vary with respect to the wake field. In Fig. 7 the effect of this is depicted with contour plots of SPL under three different stability classes. The comparison is carried out for the same incoming hub height velocity, $U_{h}=12 \mathrm{~m} / \mathrm{s}$. The results show that under stable conditions the persistence of the turbine

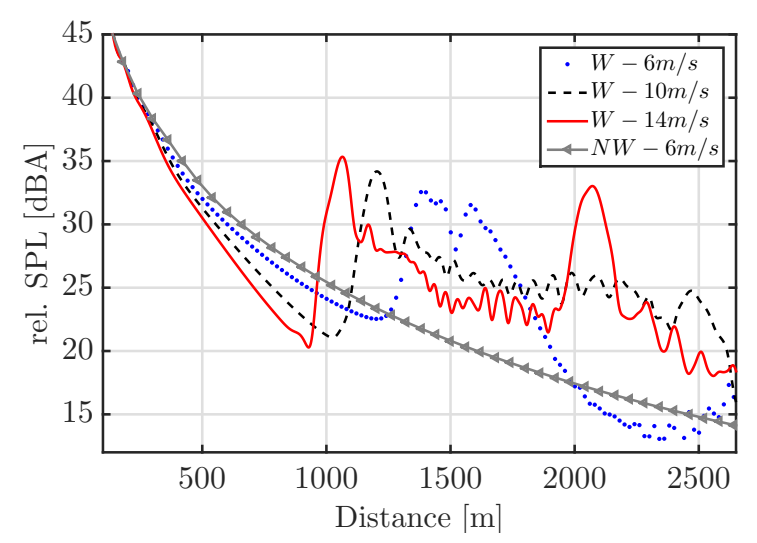

Figure 6: Sound pressure levels for a receiver at $2 \mathrm{~m}$ height, neutral boundary layer case at various incoming hub height wind speeds. Case with wake (W) and case without wake (NW).

induced velocity gradient in the wake yields a more continuous downwards refraction. Thus the region with increased sound pressure levels is widened and the start of it reaches down to a range of $1000 \mathrm{~m}$. This is also important because under stable conditions the shear of the velocity profile is higher which means that the wind speed at the ground level is considerably lower than the hub height wind speed. Therefore, the background noise at the ground level is lower which causes wind turbine sound to be more noticeable.

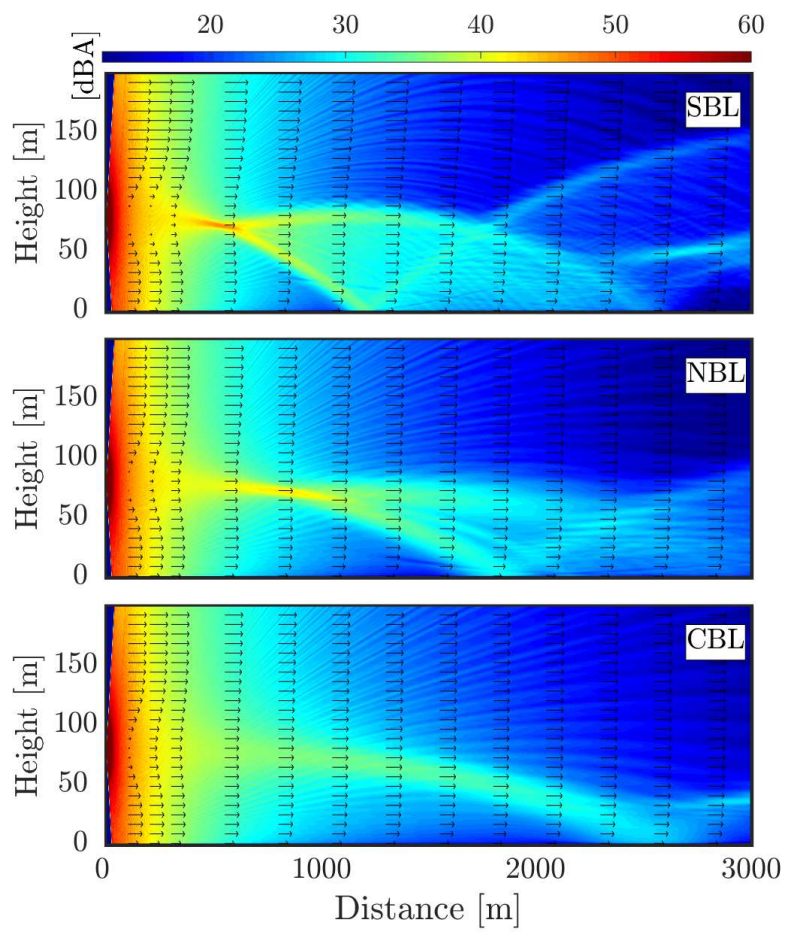

Figure 7: Contours of sound pressure levels calculated with TW-WAPE under various atmospheric stability conditions for $U_{h}=12 \mathrm{~m} / \mathrm{s}$. From top to bottom: Stable, Neutral, Convective boundary layer

Fig. 8 compares the cases with and without wake as well as 


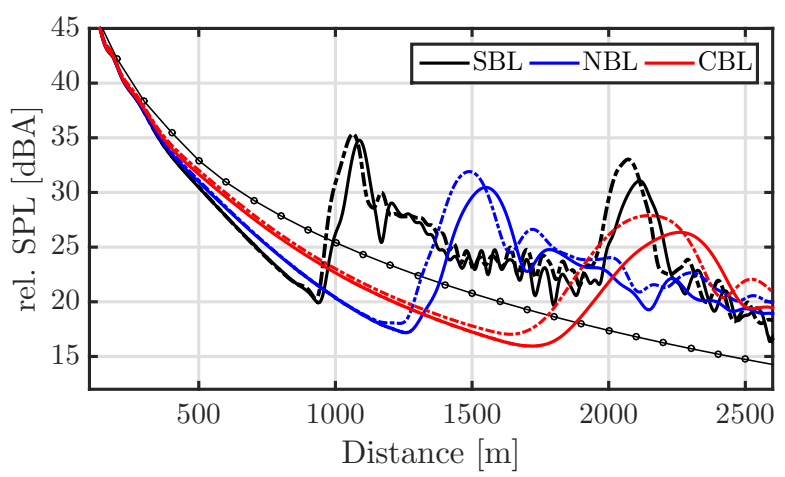

Figure 8: Sound pressure levels for a receiver at $2 \mathrm{~m}$ for $U_{h}=12 \mathrm{~m} / \mathrm{s}$. Colors represent different atmospheric stability classes. Full Line: TW-WAPE with wake. Dashed Line: WAPE with wake. Line with Markers: TW-WAPE without wake

TW-WAPE and WAPE outputs for a receiver at $2 \mathrm{~m}$ height. The results clearly indicate that the effect of wake, especially under stable atmospheric conditions is considerable. The difference in SPL reaches up to $10 \mathrm{~dB}$ for the first peak while the same value is almost $15 \mathrm{~dB}$ for the second peak under stable atmospheric condition. Another important observation is the phase difference between WAPE and TW-WAPE. As was pointed out in Blanc-Benon et al. (2001) this cumulative phase error is introduced due to the limitation of effective speed of sound approach that is inherent to WAPE. The equations derived for TW-WAPE take into account vertical and horizontal velocity components separately and speed of sound is only function of temperature. This is not the case for WAPE (scalar PE - the effective speed of sound approach). Wind speed is simply projected and added directly to the speed of sound at each space marching step. With this approach, for example a downwards refracted wave would be accelerated towards downwards. But in reality the vertical and horizontal velocity components are affecting the sound wave refraction differently than just adding it to the speed of sound. This causes a phase error that increases with distance as well as with wind speed. Here the results are not presented as a function of frequency however it was observed that at certain distances from the source the destructive and constructive interference regions change location. This results in more than 20 $\mathrm{dB}$ difference between the two methods.

\subsubsection{Effect of Source Height}

As mentioned in Sec. 2.1 the representation of a wind turbine as a single source at hub height may be misleading. Therefore simulations with different source heights are carried out. The results show different trends as a result of refraction pattern variations, see Fig. 9. A common feature of all the simulations with wake is both amplification and attenuation of SPL at various distances. This means that regardless of the source height, wake flow field redistributes the acoustic energy. If the source is located at $114 \mathrm{~m}$, the sound waves refract such that the wake induced SPL amplification is in the near field $(300 \mathrm{~m}$ $-800 \mathrm{~m}$ ). On the other hand, if the source is located at $46 \mathrm{~m}$, this amplification is observed in the far field (above $1600 \mathrm{~m}$ ). This is an interesting outcome because of two reasons; 1) as a result of continuous rotation of turbine blades the SPL increase is observed at different distances. This behaviour may help to understand the annoyance caused by amplitude modulation at the far field. 2) considering that a rotating wind turbine rotor passes through all these positions there is a cumulative effect of all source heights. This yields an overall increased SPL at all distances from the source. It is also important to mention that the study of Heimann et al. (2011) concludes that wake flow favors the sound propagation only from the upper sources towards the ground. Considering that this study is limited to 1 $\mathrm{km}$ distance this is indeed the case. However when further distances are taken into account the amplification of SPL are more considerable and mostly caused by lower sources. The effect of source location and type will be investigated further in the following section with the unsteady approach.

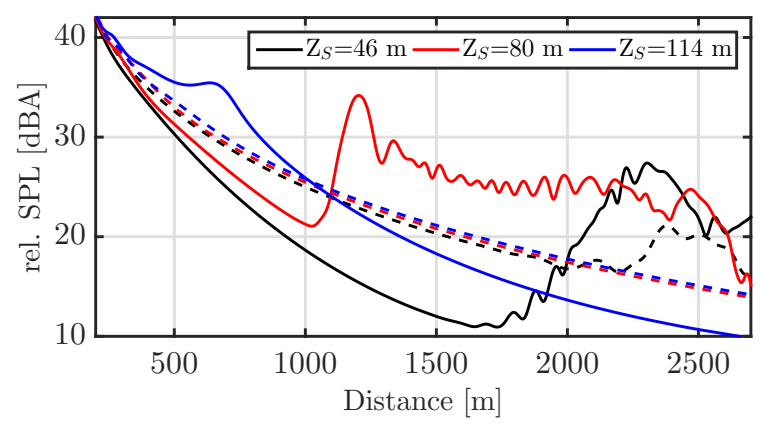

Figure 9: Sound pressure levels for a receiver at $2 \mathrm{~m}$ height for $U_{h}=10 \mathrm{~m} / \mathrm{s}$ and SBL. Comparison of range dependent (Full lines: with wake - W) and range independent (Dashed lines: without wake - NW) velocity profiles.

\subsection{Unsteady Case}

\subsubsection{Comparison of WAPE and TW-WAPE}

TW-WAPE and WAPE are compared for a five minutes long simulation. Fig. 10 shows both the mean SPL and the associated standard deviation values for a receiver at $2 \mathrm{~m}$ height. Unlike the steady flow approach there is no distinguishable phase error, neither with the moving source nor with the steady source. This can be explained via the turbulence effect on propagation. One of the major effects of turbulence on downwind sound propagation is the phase fluctuations of sound waves. This means regardless of the PE method (vector or scalar) each $P E$ run will have a 'random' phase shift. If we consider a single time step (also called a single realization) the discrepancy between the two methods is large, but once it is averaged over many realizations, the obtained results are similar.

Further assessment is carried out with the time signals obtained from both methods. Fig. 11 shows the subtracted SPL for each instant which represents the error (SPL $\left.{ }_{W A P E}-\mathrm{SPL}_{T W W A P E}\right)$. As expected, the error is increasing with distance. Around $2500 \mathrm{~m}$ and at certain instances the error reaches up to $4 \mathrm{~dB}$. 

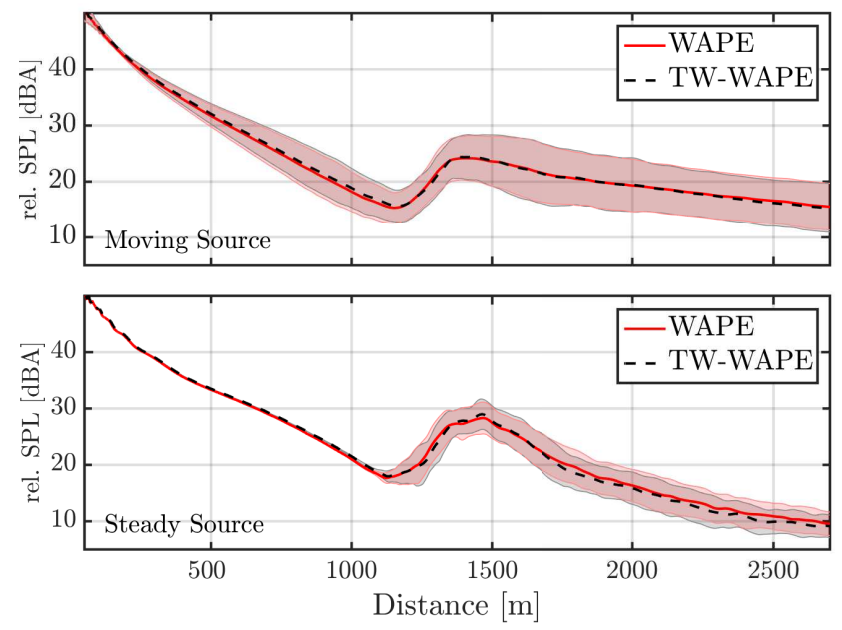

Figure 10: Sound pressure levels for a receiver at $2 \mathrm{~m}$ height obtained with two different PE methods (scalar and vector) for no turbulence incoming flow case.

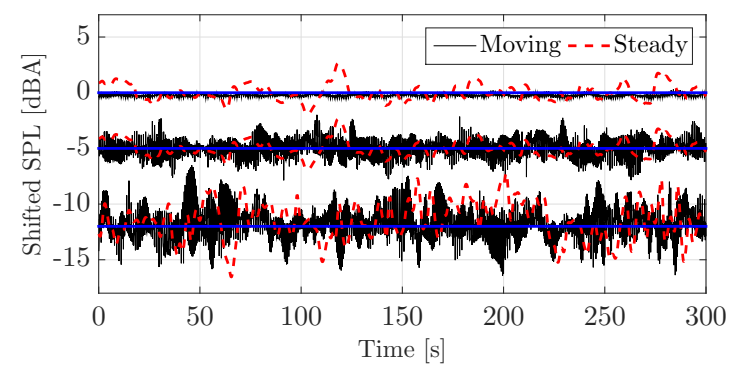

Figure 11: Difference in SPL $\left(\mathrm{SPL}_{W A P E}-\mathrm{SPL}_{T W-W A P E}\right)$ for a receiver at $2 \mathrm{~m}$ height and at three different distances. The results are shifted in y axis with the offset that blue lines represent. From top to bottom: at distances of $300 \mathrm{~m}, 1500$ $\mathrm{m}$ and $2500 \mathrm{~m}$

From these results it is concluded that the mean and standard deviation of sound pressure levels are independent of the method even though instantaneous values vary. Since the parameters of interest are restricted with those two in this study the rest of the simulations are carried out with WAPE.

\subsubsection{Effect of Wake Deficit}

In order to solely assess the effect of wake deficit dynamically three sets of simulations are carried out with moving source approach. These set of simulations are distinguished by changing the flow field input;

- LES+PE: Fully unsteady case, where every instant the flow field is updated and the source is translated up or down. This yields a twenty minutes long SPL time signal which is then averaged in time.

- Perturb+PE:Similar to the LES+PE method however, the mean wake deficit is subtracted from the flow field at each instant in time. In other words, turbine induced perturbations are superimposed to the incoming flow.
- Inflow+PE: Single logarithmic wind profile obtained from time averaged LES inflow is used along the whole propagation path (range independent, steady flow) and five PE simulations are carried out with different source heights. This yields a periodic time signal which is then averaged in time.

This comparison is conceptually similar to the steady case studies with and without wake, however for the first two methods (LES $+\mathrm{PE}$ and Perturb $+\mathrm{PE}$ ) both the flow as well as the source is dynamic which is a more realistic modelling for wind turbine noise and the atmosphere. Fig. 12 shows the differential SPL of LES+PE and Perturb+PE for three different incoming turbulence intensity, namely $0 \%, 3 \%$ and $10 \%$. Highest discrepancy is observed for the case with $0 \%(\approx 7 \mathrm{~dB})$. The constant wake deficit ducts the acoustic energy and results in a severe downwards refracting region at the far field (corresponds to the region where the wake breaks down see Fig. 2). In addition to the ground level receivers, local upward refraction results in discrepancy for all receiver heights, but the wake induced SPL amplification mostly decreases with height. All these effects are visible in a more smeared manner with increasing incoming turbulence. In other words, the start of the downwards bursting zones gets closer to the source but the overall wake induced SPL amplification decreases $(\approx 5 \mathrm{~dB})$. From this study it can be deduced that for even higher turbulence levels wake induced SPL amplification in the far field will be lower. This is also in agreement with the wake physics, since the increased incoming turbulence results in higher mixing and subsequently shorter wake. Another common feature for all cases is that the SPL discrepancy increases with distance for receivers at ground level.

Fig. 13 shows a comparison between LES+PE and Inflow+PE. The sound pressure levels for a receiver at $2 \mathrm{~m}$ height illuminates the abrupt downwards bursting zone (SPL amplification) for $0 \%$ incoming turbulence intensity case, while these regions are distributed more evenly for the other two cases. It is observed that up to a range of $500 \mathrm{~m}$ all lines collapse. This shows that atmospheric refraction effects are only visible after this distance.

\subsubsection{Effect of Source Type}

Representation of a wind turbine as a single steady point source at hub height is the conventional approach and may be an acceptable assumption for far field noise prediction. However, with the wake induced flow field the modified conditions increase the importance of source modelling type. Here this effect is investigated using moving and steady source approaches (for further explanation see Sec. 2.1). The comparison is depicted in Fig. 14. Note that, the study in Sec. 3.2.3 showed that the lower sources contribute to the SPL amplification in the far field while higher sources contribute to the near field SPL amplification. The moving source approach contains all these contributions, therefore general trend is that the SPL amplification is distributed to all distances in the domain and overall levels are mostly higher. From the two figures (Fig. 13 and Fig. 14), it can be concluded that the wake influenced sound pressure 

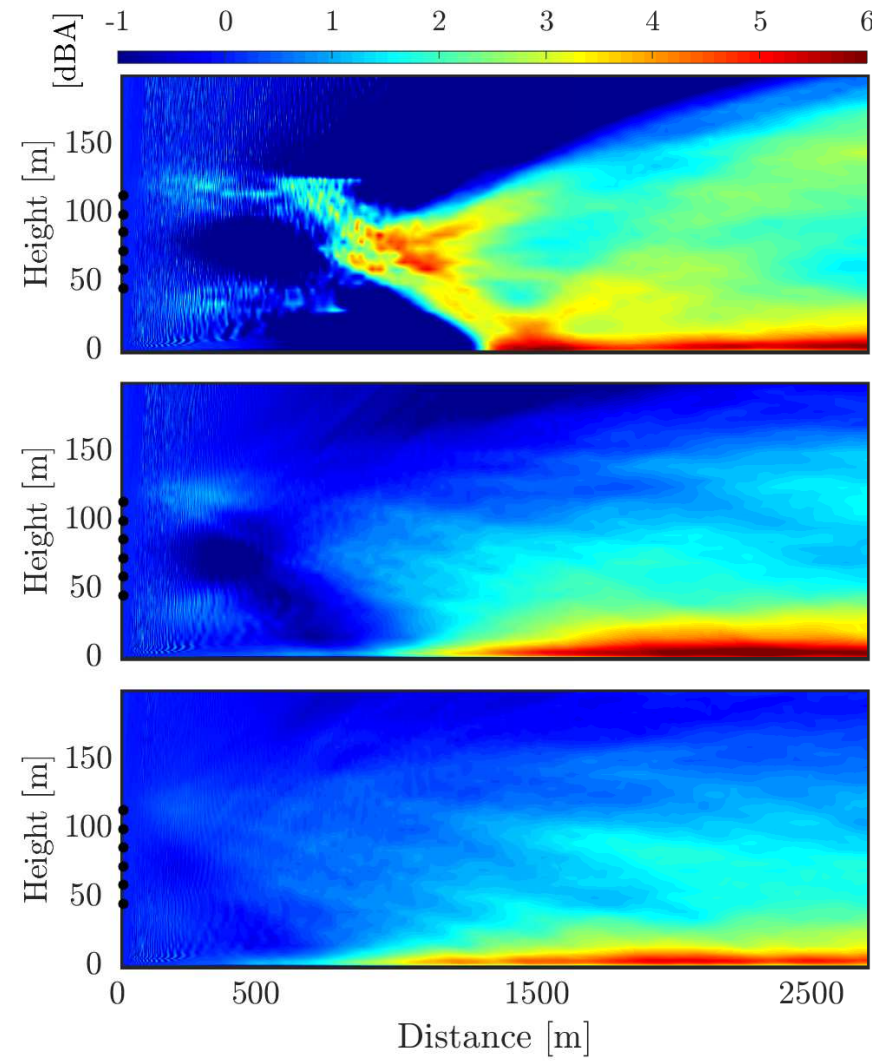

Figure 12: Contours of differential sound pressure levels $\left(\triangle_{S P L}=\mathrm{SPL}_{L E S+P E}-\mathrm{SPL}_{\text {Perturb }+P E}\right)$ for different incoming turbulence intensity. From top to bottom: T.I. $0 \%, 3 \% 10 \%$. Block dots represent the source heights

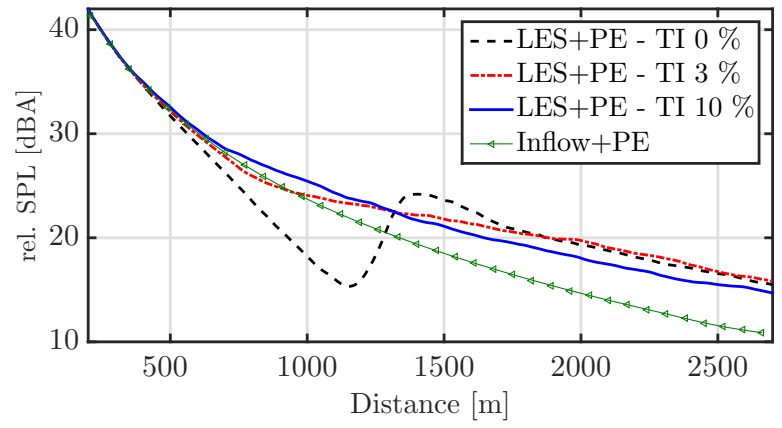

Figure 13: Sound pressure levels for a receiver at $2 \mathrm{~m}$ height, for different incoming turbulence intensity levels.

levels would be mis-predicted especially in the low turbulence cases if turbine is modelled as a single steady source at hub height.

Furthermore, the comparative study between three incoming turbulence intensity levels shows that the higher the turbulence, the less important the source type is. For example for $10 \%$ case the SPL difference between the two source modelling types is negligibly small $(\approx 1 \mathrm{~dB})$, while these values reach up to $5 \mathrm{~dB}$ for both cases with $0 \%$ and $3 \%$. For the case with $0 \%$ incoming turbulence intensity the abrupt increase of SPL due to severe downward refraction is seen with both source types. However, the amplification is lower for moving source at certain distances. These observations are in agreement with the wake physics as well. Higher incoming turbulence results in more mixing and shorter wake. Hence, refraction of sound waves due to wake induced flow field is not so influential. Subsequently the source position or modelling type becomes less important.
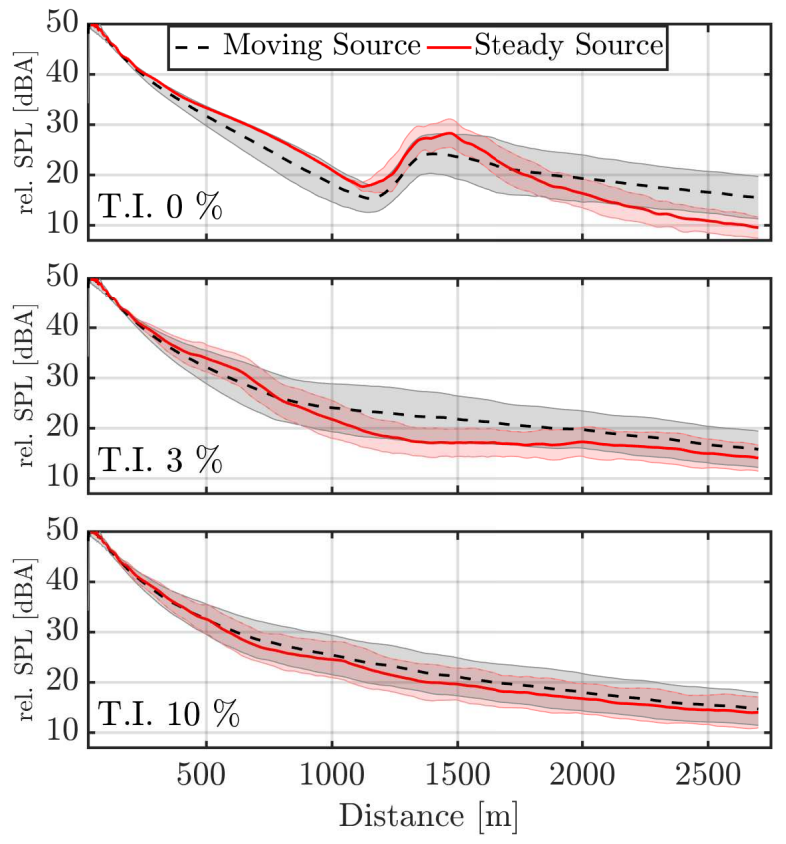

Figure 14: Sound pressure levels for a receiver at $2 \mathrm{~m}$ height, for different incoming turbulence intensity levels and source types. Shaded regions represent the standard deviation.

The shaded regions in Fig. 14 represent the standard deviation of SPL. For an easier comparison these values are shown in Fig. 15. In the near field a clear effect of source type is seen. For all flow cases with moving source a linear relationship between distance and standard deviation is detected up to a certain point, after which the values for both source types collapse. This distance varies depending on the incoming turbulence intensity. These values are $180 \mathrm{~m}$ and $140 \mathrm{~m}$ for $3 \%$ and $10 \%$, respectively. The higher the turbulence intensity, the earlier the source type loses its effect. Afterwards the fluctuations due to flow dynamics rather than the source become more important.

At the far field (above $700 \mathrm{~m}$ ) there is almost no difference between two source types for the $10 \%$ case. However, there is a certain discrepancy for $3 \%$ case which can be explained with the longer wake. Due to more severe and persistent local downwards refracting regions the source location becomes a more important issue for the $3 \%$ case. Sound emitted from the lower sources with wake deficit results in higher contribution to downwards bursting zones. And with the movement of the source the receiver at certain distances goes in and out of these increased SPL regions. This shows that the source type is 
important for large distances under low turbulence intensity.

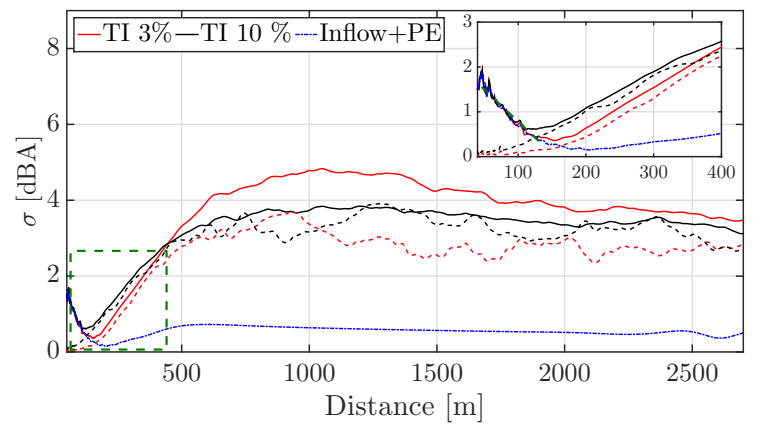

Figure 15: Standard deviation $(\sigma)$ of SPL for a receiver at $2 \mathrm{~m}$ height under various flow conditions and source modelling techniques. Dashed lines: Steady source. Full lines: Moving source.

\subsection{Comparison between Steady and Unsteady Case}

This part of the paper is devoted to the comparison among different flow field input methods. The simulations are carried out with the moving source approach and three different ways are used to feed the flow to the acoustic simulations;

- LES+PE: Fully unsteady case, where the flow field is updated every instant and the source is translated up and down. This yields a twenty minutes long SPL time signal which is then averaged in time.

- MeanLES+PE: In total only five simulations are carried out for five different source heights along the rotor. The flow field is obtained via time averaging the LES results and remains unchanged for all simulations. This yields a periodic time signal which is then averaged in time.

- Analytical Wake+PE (AW+PE): Similar to the method MeanLES+PE, this time the flow field is obtained from superposition of the inflow wind profile with an analytical wake model where the wake growth parameter $\left(\mathrm{k}^{*}\right)$ is tuned with respect to the LES results. This yields a periodic time signal which is then averaged in time.

Fig. 16 shows the comparison of time averaged SPL for all three methods and incoming turbulence intensities. There is a clear discrepancy between AW+PE and the other two methods. The difference decreases with increasing turbulence intensity however it is observed that the AW+PE method underestimates the SPL along the whole propagation path for all cases. For the laminar inflow case (incoming turbulence intensity $0 \%$ ) SPL amplification around the range of $1250 \mathrm{~m}$ is captured to a certain extend. Nevertheless this increase is not as abrupt as in LES+PE or MeanLES+PE. This is because analytical wake model is a far field model. This means the method does not capture the flow characteristics in the near field and the PE method is sensitive to the flow input. In order to overcome this issue a refined wake model that is capable of capturing the near wake flow field more accurately can be used.

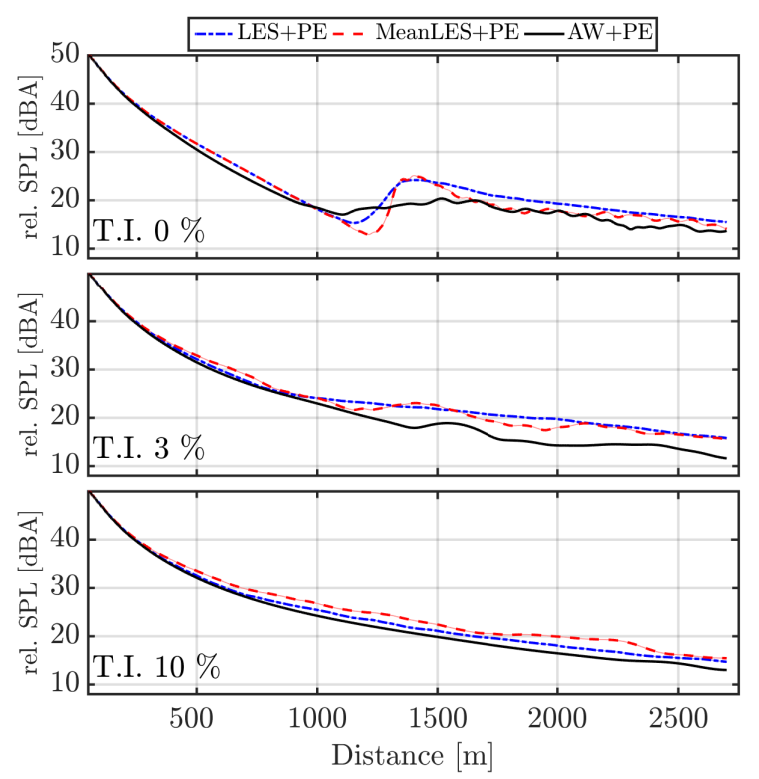

Figure 16: Sound pressure levels for a receiver at $2 \mathrm{~m}$ height for three different incoming turbulence intensity and flow field input methods.

Another important observation is that the discrepancy between averaged SPL obtained from LES+PE and MeanLES $+\mathrm{PE}$ is not very significant. They both follow a similar trend and the differences in averaged SPL do not exceed $3 \mathrm{~dB}$. This means the wind turbine induced turbulence does not influence the downwind sound propagation much.

Moreover, time signals of sound pressure levels are investigated. Fig. 17 shows the signals for a receiver height of $2 \mathrm{~m}$ at three different location, namely $100 \mathrm{~m}, 700 \mathrm{~m}$ and $2500 \mathrm{~m}$ also referred to as near field, mid-field and far field in this section. It is observed that in the near field $(100 \mathrm{~m})$ for all incoming TI the sound pressure levels are modulated periodically as a result of the moving source. Even though certain peaks are visible for the SPL time signal obtained with LES+PE the peak to trough ratio and the mean SPL values are same for all three methods. This implies that in the near field the source is the dominant parameter, which governs the sound pressure levels rather than the flow input.

In the mid-field $(700 \mathrm{~m})$ time signal of all methods collapse again for the laminar inflow case (incoming T.I. $0 \%$ ). At this distance for this case the wake breakdown has not taken place therefore the moving source is again the only cause for periodic modulation that is observed for all methods. However for the other cases with higher incoming TI (3\% and $10 \%$ ) even though the mean SPL values are very close to each other for all three methods the behaviour of the signals are different. $\mathrm{AW}+\mathrm{PE}$ and MeanLES $+\mathrm{PE}$ shows periodic modulation since there is no changes in the flow. LES+PE has both the modulation due to changing source height as well as the turbulent flow field. This yields higher peak to trough values at various instances in time. For example maximum values reach up to 23 $\mathrm{dB}$ for the case with incoming TI $3 \%$ while this is maximum $18 \mathrm{~dB}$ for the TI $10 \%$ case. Note that the standard deviation of LES+PE for $3 \%$ case is higher than $10 \%$ case. This ob- 
servation was also underlined in sec 3.3 .3 (see Fig. 15). This is because the ducted acoustic energy due to persistent wake is refracted downwards and reaches different distances at each instant in time as the source moves up and down. As a result of this the time signal at a certain receiver point has higher standard deviation.

In the far field $(2500 \mathrm{~m})$ time signals of $\mathrm{AW}+\mathrm{PE}$ and MeanLES +PE still show a periodic modulation. However the peak to trough values decrease with increasing turbulence (i.e. $16 \mathrm{~dB}$, $12 \mathrm{~dB}$ and $5 \mathrm{~dB}$ for $0 \%, 3 \%$ and $10 \%$ respectively). From this observation it can be concluded that if the wake is short enough (e.g. very high turbulence) the fluctuations that are seen in the far field due to the moving source will disappear. The results of LES+PE also supports this argument. They show a similar trend in terms of standard deviation. Remembering again the study in sec 3.3.3 the standard deviation of moving and steady source collapse on the same line at this distance (above 2300 $\mathrm{m})$.

So far the comparison is restricted with the summed SPL values that are calculated using Eq. 2. For further investigation frequency dependent spectra for a receiver height of $2 \mathrm{~m}$ are depicted in Fig. 18. From summed and averaged SPL comparison it was clear that the AW+PE performs poorly and the results do not match the other two methods. The comparisons of spectra also shows that $\mathrm{AW}+\mathrm{PE}$ does not capture the general trend as LES+PE or MeanLES+PE. Higher differences are observed for the frequencies over $300 \mathrm{~Hz}$. The frequency dependent comparisons between LES+PE and MeanLES+PE has interesting output. MeanLES +PE captures the general trend however LES+PE has a more smeared distribution of SPL over the whole propagation path which can be explained by the phase shifts caused by turbulence. This can not be captured by running only five simulations without changing the flow field. 

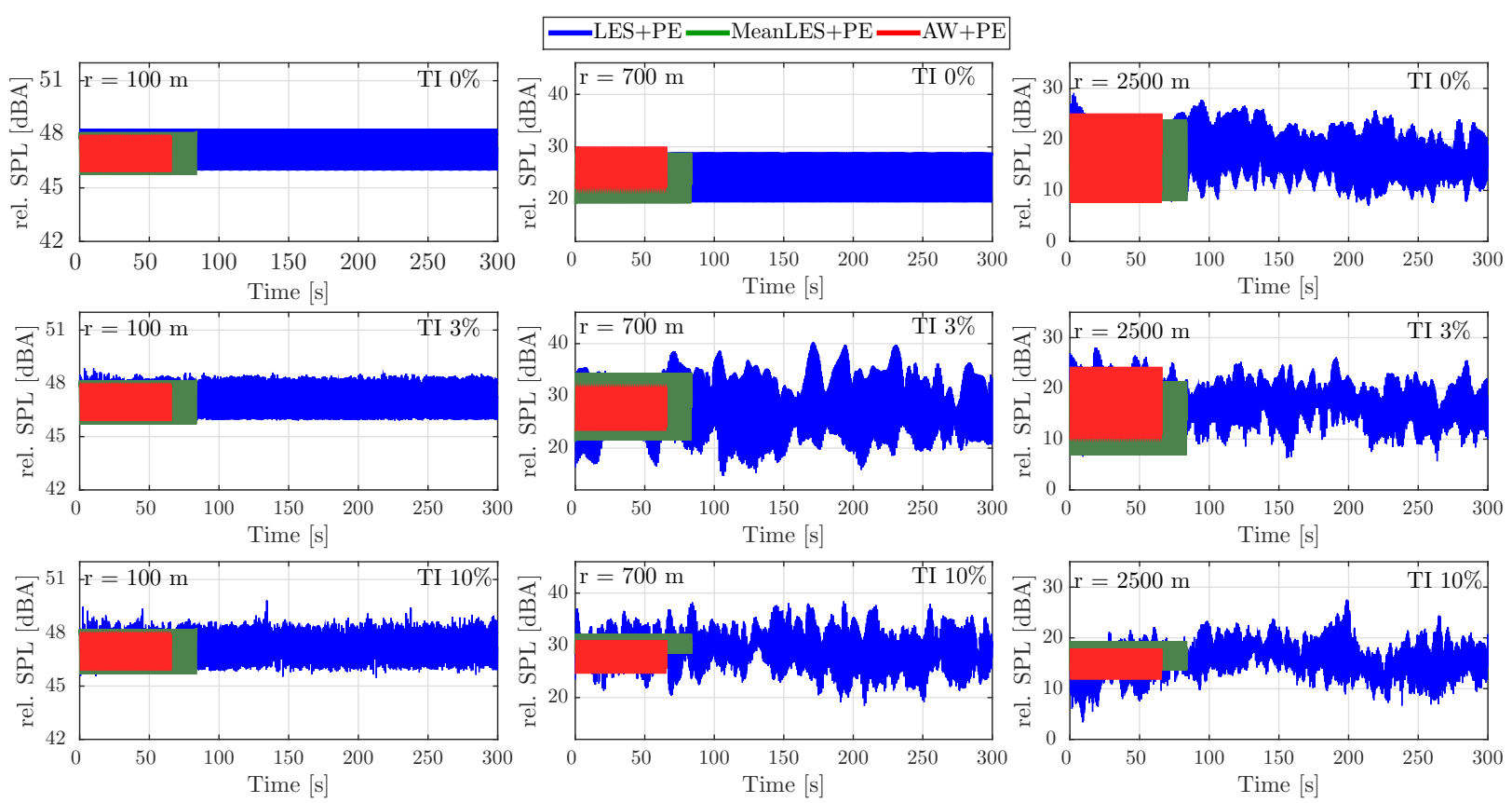

Figure 17: Time signal of SPL for a receiver at $2 \mathrm{~m}$ height and different distances for cases with different incoming turbulence intensities. Three different flow field input methods are used.
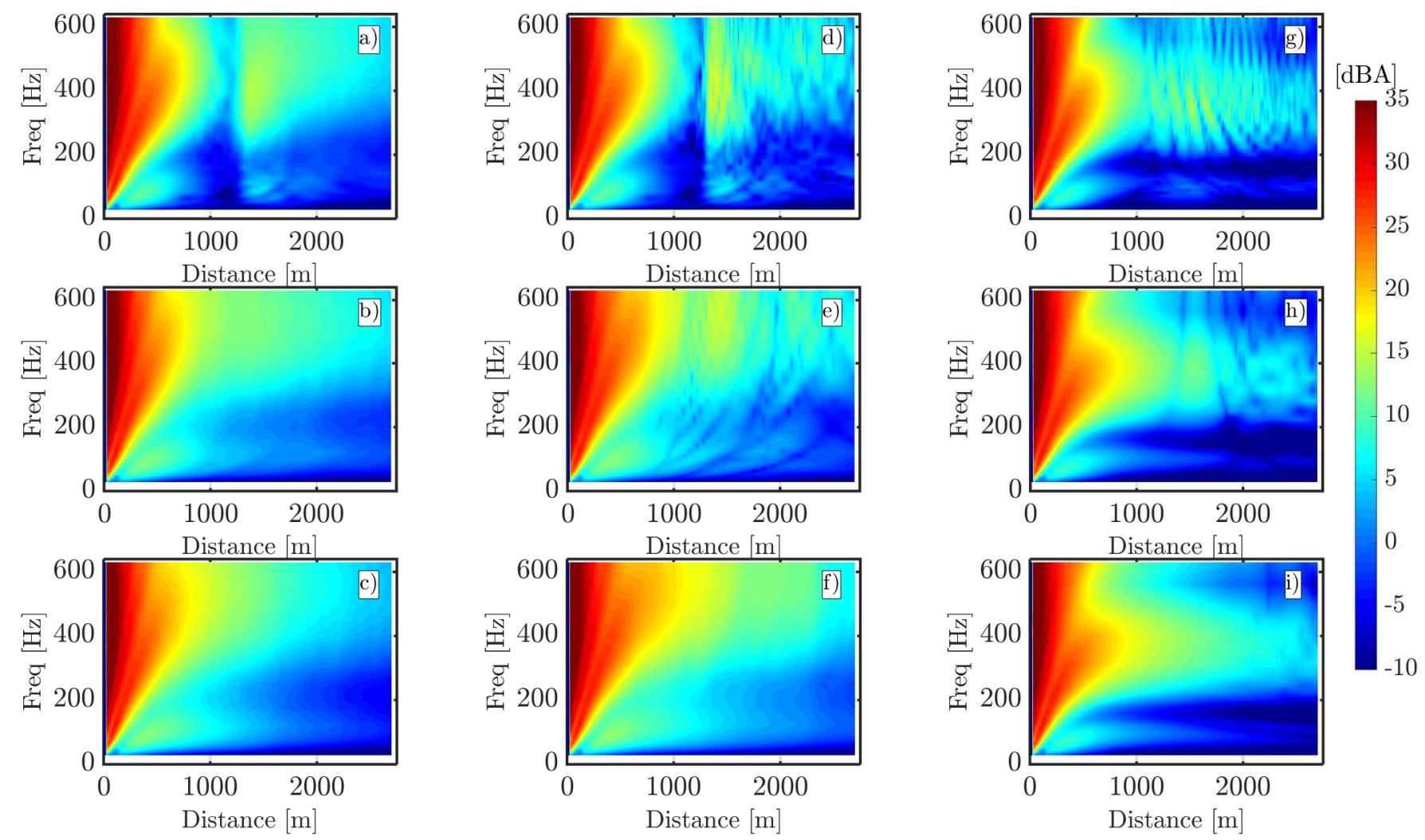

Figure 18: Frequency dependent (1/3-octave band lower, centre and upper limit) SPL for a receiver at $2 \mathrm{~m}$ height for three different incoming turbulence intensity and flow field input methods. a) LES+PE - TI $0 \%$ b) LES+PE - TI $3 \%$ c) LES+PE - TI $10 \%$ d) MeanLES+PE - TI $0 \%$ e) MeanLES+PE - TI $3 \%$ f) MeanLES+PE - TI $10 \%$ g) AW+PE - TI $0 \%$ h) AW+PE - TI $3 \%$ i) AW+PE - TI $10 \%$ 


\section{Discussion}

From this study it is clear that the acoustic energy is redistributed downwind from wind turbine, due to the wake-induced flow field. The main parameter is the persistence of the wake, particularly in stable atmospheric conditions: there the wake is long, considerably high SPL amplification is observed in the far field. There are certain shortcomings of this study that may prevent generalization to some wake cases. First of all, even though the steady case investigates three different incoming wind profiles, under more extreme conditions (i.e. friction velocity $u_{*}=8 \mathrm{~m} / \mathrm{s}$ ) it is possible that the wake flow may result in SPL attenuation also in far field as it was pointed out in Lee et al. (2015). Nevertheless, the cases considered in this study covers the atmospheric conditions that are commonly encountered and a more detailed study was also carried out by the authors that investigates the effect of shear and turbulence on both downwind and upwind propagation from a wind turbine Barlas et al. (2016). Additionally, it may be argued that the moving source approach is a limitation of the present study. Wind turbine blades rotate in and out of the 2D PE domain instead of only along one axis. However, the further the receiver, the more realistic it becomes to model the rotor as a single source that moves up and down. This is a source modelling technique that captures most of the dynamics, such as amplitude modulation due to blade passage.

The unsteady approach which couples PE with LES for wind turbines is state of the art. However, as comprehensively investigated in Cotté and Blanc-Benon (2007), the smallest turbulent scales should be on the order of the acoustic wavelength for accurate modelling of the turbulence effect on scattering. This condition is not met considering the highest frequency resolved using $\mathrm{PE}$ is $630 \mathrm{~Hz}(\lambda \approx 0.5 \mathrm{~m})$ and the LES spatial resolution is $2.5 \mathrm{~m}$ for all directions. On the other hand the required resolution is computationally so demanding that a fully resolved coupling is not considered in this study. Last, it is important to point out that the current study is limited to flat terrain and homogeneous ground impedance. These are important parameters that cause sound waves to reflect differently. Further investigations should be carried out to see if the dynamic wake effects are larger for sound propagation over complex terrain and inhomogeneous ground impedance.

\section{Conclusion}

It is known that different atmospheric conditions result in various acoustic refraction patterns. When the source is a wind turbine, the correct representation of the flow and source itself becomes even more important because of the constant variations in the source strength and flow modifications around wind turbines. In this study, both steady and unsteady approaches were employed using a 2D PE method coupled to flow fields generated by an analytical wake model and AL/LES. The main findings are:

- Sound waves go through local upward and downward refracting regions, as a result of wake deficit behind a wind turbine. This means acoustic simulations without the wake can not properly estimate the far field noise. This is valid for all atmospheric stability conditions, incoming wind speeds and source heights that have been investigated in this study.

- It is observed that during stable atmospheric conditions, which result in long and persistent wakes, far field noise can be severely under-predicted if the correct flow field is not used.

- By comparing two different source modelling techniques (moving and steady) coupled with LES it is concluded that the effect of the moving source in far field (beyond $700 \mathrm{~m}$ $\approx 9 \mathrm{D})$ diminishes with increasing incoming turbulence intensity. On the other hand, at the near field the effect of the moving source is always felt. The standard deviation values of SPL with steady and moving source collapse first after $140 \mathrm{~m}(\approx 1.8 \mathrm{D})$ for $10 \%$, while this value is approximately $180 \mathrm{~m}(\approx 2.3 \mathrm{D})$ for $3 \%$.

- For a single realization of the 2D PE there is a cumulative phase error caused by intrinsic assumption of effective sound speed approach (scalar PE - WAPE). However, the discrepancy between vector and scalar PE using the time dependent LES results, is negligibly small. This is possibly due to the random phase shifts at each instant in time caused by turbulent flow which diminish the error when averaged in time.

- The mean SPL behind a wind turbine can be predicted with time averaged input (MeanLES+PE) almost as accurately as time dependent input (max. $3 \mathrm{~dB}$ ). However, the comparison with PE using an analytical wake model did not yield satisfactory results. This is because analytical wake models do not capture the near wake characteristics of the flow. Subsequently the sound waves are not refracted in the same way as LES results.

\section{Acknowledgements}

The work has been funded by the GreenTech Wind project, a collaboration of the EuroTech Universities. Furthermore, the proprietary data for Vestas' NM80 turbine has been used.

\section{References}

Aagaard Madsen, H., Bak, C., Schmidt Paulsen, U., Gaunaa, M., Fuglsang, P., Romblad, J., Olesen, N., Enevoldsen, P., Laursen, J., Jensen, L., 2010. The DAN-AERO MW Experiments. Denmark. Forskningscenter Ris $\emptyset$. Ris $\emptyset$-R. Danmarks Tekniske Universitet, Ris $\emptyset$ Nationallaboratoriet for Bæredygtig Energi.

Abkar, M., Porte-Agel, F., 2014. The effect of atmospheric stability on windturbine wakes: A large-eddy simulation study. Journal of Physics: Conference Series 524 (1), 012138-.

URL http: //stacks . iop.org/1742-6596/524/i=1/a=012138

Barlas, E., Zhu, W. J., Shen, W. Z., Andersen, S. J., 2016. Wind turbine noise propagation modeling : An unsteady approach. In: Journal of Physics: Conference Series, Volume 753, The Science of Making Torque from Wind 2016. 
Bastankhah, M., Porte-Agel, F., Oct. 2014. A new analytical model for wind-turbine wakes. Renewable Energy 70, 116-123.

URL http://www.sciencedirect.com/science/article/pii/ S0960148114000317

Blanc-Benon, P., Dallois, L., Juve, D., 2001. Long range sound propagation in a turbulent atmosphere within the parabolic approximation. Acta Acustica united with Acustica 87 (6), 659-669.

Cotté, B., Blanc-Benon, P., 2007. Estimates of the relevant turbulent scales for acoustic propagation in an upward refracting atmosphere. Acta Acustica united with Acustica 93 (6), 944-958.

Dallois, L., Blanc-Benon, P., Juve, D., 2001. A wide-angle parabolic equation for acoustic waves in inhomogeneous moving media: Applications to atmospheric sound propagation. Journal of Computational Acoustics 9 (02), 477-494.

Delany, M. E., Bazley, E. M., 1970. Acoustical properties of fibrous absorbent materials. Applied Acoustics (3), 105-116.

Heimann, D., Kaasler, Y., Gross, G., 2011. The wake of a wind turbine and its influence on sound propagation. Meteorologische Zeitschrift, Vol. 20, No. 4, 449-460.

Kelly, M., Gryning, S.-E., 2010. Long-term mean wind profiles based on similarity theory. Boundary-Layer Meteorol 136 (3), 377-390.

Lee, S., Lee, D., Honhoff, S., 2015. Prediction of far-field wind turbine noise propagation with parabolic equation. In: 21st AIAA/CEAS Aeroacoustics Conference, Dallas, Texas.

Mann, J., 1998. Wind field simulation. Probabilistic engineering mechanics 13 (4), 269-282.

Michelsen, J., 1992. Basis 3d-a platform for development of multiblock pde solvers, afm 92-05. Tech. rep., Technical University of Denmark.

Oerlemans, S., Sijtsmaa, P., Mendez Lopez, B., 2007. Location and quantification of noise sources on a wind turbine. Journal of Sound and Vibration $299,869-883$

URL http://www.sciencedirect.com/science/article/pii/ S0022460X06006316

Ostashev, V., Juve, D., Blanc-Benon, P., 1997. Derivation of a wide-angle parabolic equation for sound waves in inhomogeneous moving media. Acta Acustica (Stuttgart) 83 (3), 455-460.

Salomons, E. M., 2001. Computational Atmospheric Acoustics. Springer Science + Business Media B. V.

Sørensen, J., Mikkelsen, R., Henningson, D., Ivanell, S., Sarmast, S., Andersen, S., 2015. Simulation of wind turbine wakes using the actuator line technique. Phil. Trans. R. Soc. A 373 (2035).

Sørensen, J. N., Shen, W. Z., May 2002. Numerical modeling of wind turbine wakes. Journal of Fluids Engineering 124 (2), 393-399.

URL http: //dx .doi .org/10.1115/1.1471361

Sørensen, N. N., 1995. General purpose flow solver applied to flow over hills ph.d. thesis. Ph.D. thesis, Technical University of Denmark.

West, M., Gilbert, K., Sack, R., 1992. A tutorial on the parabolic equation (pe) model used for long range sound propagation in the atmosphere. Applied Acoustics 37 (1), 31-49.

Wilson, D. K., Noble, J. M., Coleman, M. A., 2003. Sound propagation in the nocturnal boundary layer. Journal of the atmospheric sciences 60 (20), 2473-2486.

Øye, S., 1996. Flex4 simulation of wind turbine dynamics.

Zhu, W. J., Heilskov, N., Shen, W. Z., Sorensen, J. N., Jun. 2005. Modeling of aerodynamically generated noise from wind turbines. Journal of Solar Energy Engineering 127 (4), 517-528.

URL http://dx.doi.org/10.1115/1.2035700 